\title{
Twin Gas Jet-assisted Pulsed Green Laser Scribing of Sapphire Substrate
}

\author{
X.Z. Xie, Z.Q. Luo, X. Wei, F.L. Zhang, W. Hu and Q.L. Ren \\ School of Eletromechanical Engineering, Guangdong University of Technology, Guangzhou, \\ Guangdong 510006 , China \\ E-mail:xiaozhuxie@gdut.edu.cn
}

\begin{abstract}
Sapphire is an important material for LED substrates. Because of its unique physical property, sapphire is strongly resistant to wet and dry chemical etching. Laser-based scribing techniques have a good prospect on sapphire scribing application. During the laser scribing, the assisting gas significantly affects the scribing depth, width and quality. A pulsed laser with a wavelength of $532 \mathrm{~nm}$ is used to scribe the sapphire substrate by twin assisting gas impinging both in co-axis and off-axis. This process is simulated by a computational fluid dynamics code, FLUENT. During the simulation, inlet pressure of co-axial nozzle and off-axial nozzle are varied at different intersection angle. By analyzing the static pressure and flow velocity at different inlet pressure and intersection angle, optimal laser processing parameters are obtained to improve the groove quality and reduce the groove width. Experiments are carried out to testify the simulation result. The result of both the simulation and experiment show that when laser pulse energy is $150 \mu \mathrm{J}$ with co-axial inlet pressure 2 bar, offaxial inlet pressure 2 3 bar and intersection angle $70^{\circ}$, a better groove quality and an optimal groove size can be obtained.
\end{abstract}

DOI: $10.2961 / \mathrm{jlmn} .2016 .02 .0006$

Keywords: assisting gas, laser scribing, sapphire, flow field, FLUENT

\section{Introduction}

Sapphire has been widely used in industry, national defense and scientific research. And it is an important material for LED substrates and optical windows for its unique physical property and strong capacity to resist wet and dry chemical etching [1]. Since the sapphire is hard and fragile, it is difficult to fabricate by traditional mechanical machining. Laserbased scribing, being a non-contact process is a suitable and efficient method for precision machining of sapphire without the problems of mechanical damage and tool wear. Sapphire scribing has a great impact on the yields of the component and packaging efficiency. During the laser scribing, redeposition layer around the scribing groove increases the groove width. That is why reducing and eliminating the fused deposition is the key to improve the groove quality and increase components yields. The assisting gas has been widely used for removing the melting material in laser metal cutting and drilling. Efficiency and quality are closely related to gas pressure, nozzle geometry and stand-off distance. By adjusting these parameters, the generation of normal standoff shock which reduces the total pressure loss can be avoided [2]. W O'Nell [3] indicates that in laser metal cutting, the angle between the laser beam and the off-axial gas jet determines the maximum groove depth \& width and the generation of a suitable clockwise rotating vortex may aid the removal of molten material. Sezer [4] investigates the influence of angle in twin gas jet-assisted laser drilling and analyzes the flow field at different angles. Mai [5] studies the phenomena of shock wave induced by a supersonic impinging jet emanating from a straight nozzle onto a substrate with varying inclined angles by numerical simulation and experiment. He points out that if the total pressure is high, a Mach shock disk is formed and the gas pressure drops dramatically beyond the shock disk. Melhem [6] calculates the skin friction along the kerf surface for four average jet velocities at the nozzle exit and two kerf wall wedge angles. It indicates that the skin fiction decreases along the kerf surface. B Tirumala Rao [7] points out that if we want to achieve the minimum melt film thickness on the cut surface, laser cutting should be performed at optimum cutting velocity and maximum gas pressure so that laminar gas flow is sustained in the entire kerf depth. Jin-chen Hsu [8] demonstrates that the use of the intermittent gas jets can effectively increase the material removal rate and reduce the consumption of assisting gas. As a result, in laser metal processing the main factors are standoff distance, intersection angle and inlet pressure. Especially, inlet pressure is the most important factor because an excessive inlet pressure induces the generation of normal standoff shock which will deteriorate the processing quality.

On the other hand, other researchers promote the melt remove ability of assisting gas by improving supersonic nozzle structure. Guo [9, 10] makes an 
analysis of relationship between nozzle structure and dynamics characteristic of flow field. Qiu [11] establishes the turbulence model of subsonic convergent nozzle, analyzes the influence law of gas dynamics performance about jet flow affected by the nozzle structure, constructs a three-dimensional symmetry model of laser cutting impinging flow and studies the influence on the gas dynamics performance by laser cutting parameters in kerf. Due to the difficulty of processing the supersonic nozzle, modifying the shape and parameters inside the nozzle to improve the function of gas dynamics in the process of laser cutting costs too much. Therefore, the main method to optimize the dynamic performance of the laser cutting process is to examine the influence of different assisted gas parameter on the gas flow field.

Assisting gas is not only used in metal material laser processing, but also used in other materials. Sulaiman [12] investigates that high assisting gas pressure results in slightly large kerf width but small out-of-flatness and kerf width ratios in Kevlar laminate laser cutting. Elhadj S [13] uses an assisting gas in lowering treatment temperatures and changing interfacial and bulk chemistry to limit capillarydriven flow in laser silica ablating.

In this paper we simulate the assisting gas flow by computation fluid dynamics software FLUENT based on the Navier-Stokes equation of compressible gas, so as to investigate the influence of different processing parameters on the flow field of assisting gas. Combing with experiments we study how inlet pressure and intersection angle of co-axial and offaxial jet nozzle affect the depth and width of the groove, so as to obtain optimum laser processing parameters to scribe a deep and narrow groove with good quality.

\section{Theoretical modeling}

\subsection{Basic equation of turbulent flow}

During the laser scribing, the interaction of assisting gas and workpiece surface can be simulated by symmetrical jet impact model, which is based on steady compressible Reynolds average Navier-Stokes equation [14].

Reynolds average Navier-Stokes equation:

Conservation of mass:

$$
\frac{\partial \rho}{\partial \tau}+\frac{\partial}{\partial x_{i}}\left(\rho u_{i}\right)=0
$$

Where $\rho$ is the gas density and $u_{i}$ is the gas velocity in the direction $i$.

Conservation of momentum

$$
\begin{aligned}
& \frac{\partial}{\partial \tau}\left(\rho u_{i}\right)+\frac{\partial}{\partial x_{j}}\left(\rho u_{i} u_{j}\right)=\frac{\partial p}{\partial x_{i}}+ \\
& \frac{\partial}{\partial x_{i}}\left[\mu_{\mathrm{ef}}\left(\frac{\partial u_{i}}{\partial x_{j}}+\frac{\partial u_{j}}{\partial x_{i}}-\frac{2}{3} \delta_{i j} \frac{\partial u_{i}}{\partial x_{j}}\right)\right]+\frac{\partial}{\partial x_{j}}\left(-\rho u_{i}^{\prime} u_{j}^{\prime}\right)
\end{aligned}
$$

Where $p$ is the gas pressure, $\mu_{\mathrm{ef}}$ is the effective viscosity and $u_{j}$ is the gas velocity in the direction $j$.

Turbulence model:

RNG k- $\varepsilon$ is adopted because a big gap occurs by using standard $\mathrm{k}-\varepsilon$ equation in simulating the impingement of assisting gas $[15,16]$. Where $\mathrm{k}$ is the turbulent kinetic energy and $\varepsilon$ is the dissipation rate of the turbulent kinetic energy in the RNG k- $\varepsilon$ turbulence model. The values of $\mathrm{k}$ and $\varepsilon$ can be obtained from Esq.(3) and (4).

$$
\begin{aligned}
& \rho \frac{\mathrm{D} k}{D \tau}=\frac{\partial}{\partial x_{i}}\left[\alpha_{k} \mu_{e f} f \frac{\partial k}{\partial x_{i}}\right]+G_{k}-\rho \varepsilon-Y_{M} \\
& \rho \frac{\mathrm{D} k}{D \tau}=\frac{\partial}{\partial x_{i}}\left[\alpha_{k} \mu_{e f} f \frac{\partial k}{\partial x_{i}}\right]+\mathrm{C}_{1 \varepsilon} \frac{\varepsilon}{k} G_{k}-C_{2 \varepsilon}^{*} \rho \frac{\varepsilon^{2}}{k}
\end{aligned}
$$

Where $\alpha_{\mathrm{k}}$ and $\alpha_{\varepsilon}$ are the effective prandtl numbers of $\mathrm{k}$ and $\varepsilon$ respectively. Here both $\alpha_{\varepsilon}$ and $\alpha_{\mathrm{k}}$ are 383 . $\mathrm{G}_{\mathrm{k}}$ is the the generation term of turbulent kinetic energy,

$G_{k}=-\rho \overline{u_{i}^{\prime} u_{j}^{\prime}} \frac{\partial u_{j}}{\partial x_{i}}$

According to Bousssinesq hypothesis,

$\mathrm{G}_{\mathrm{k}}=\mu_{\tau} S^{2}$

Where $\mu_{\tau}=\rho C_{\mu} \frac{k^{2}}{\varepsilon}, \quad S=\sqrt{2 S_{i j} S_{i j}}$

$S_{i j}=\frac{1}{2}\left(\frac{\partial u_{i}}{\partial x_{j}}+\frac{\partial u_{j}}{\partial x_{i}}\right)$

$Y_{M}$ is the correction term of compressible gas.

$$
Y_{M}=\rho \varepsilon 2 M_{\tau}^{2}
$$

$$
\begin{aligned}
& \text { Where } M_{\tau}=\sqrt{\frac{k}{\alpha^{2}}}, \alpha=\sqrt{\gamma R T}, \\
& C_{2 \varepsilon}^{*} \equiv C_{2 \varepsilon}+\frac{C_{\mu} \rho \eta^{3}\left(1-\eta / \eta_{0}\right)}{1+\beta \eta^{3}}
\end{aligned}
$$

Where $\eta=S k / \varepsilon, S$ is the tensor module of tensor rate, $C_{1 \varepsilon}=1.42, \quad C_{2 \varepsilon}=1.68, \quad \mathrm{C}_{\mu}=0.0845, \quad \eta_{0}=4.38$, $\beta=0.012$.

Considering vortex flow in the average flow, RNG $\mathrm{k}-\varepsilon$ equations have corrected the turbulent viscosity. By addicting a correction term in the $\varepsilon$ equation, stain rate of the mainstream appears. Therefore, the generation term in RNG k- $\varepsilon$ equations not only connects with the flow condition but also is the function of spatial coordinates in the simulation case. RNG $\mathrm{k}-\varepsilon$ equations is widely used in calculating parameter of nozzle jet flow because it is better dealing with high strain rate flow which has a 
large curvature degree steam line. Taking the accuracy of simulation into account, the RNG k- $\varepsilon$ equations are selected to build turbulence model.

\subsection{Boundary condition and meshing}

In the simulation of the supersonic assisting jets impinging on the sapphire substrate, the computation domain is selected as the region between the nozzle and the substrate shown in Fig.1. There is a co-axial jet nozzle and an off-axial jet nozzle in the numerical model, where center line of co-axial nozzle is perpendicular to the surface of workpiece and the intersection angle between co-axial nozzle and offaxial nozzle $(\theta)$ is varying from $5^{\circ}$ to $85^{\circ}$. Stand-off distances of both co-axial nozzle and off-nozzle are selected as $5 \mathrm{~mm}$ because the pressure impinging on the workpiece surface is in the second high pressure area at this distance [17]. Exit diameter of co-axial nozzle is $2 \mathrm{~mm}$ and exit form of off-axial nozzle is rectangle with size of $10 \mathrm{~mm} \times 1 \mathrm{~mm}$. Compressible air is used as the assisting gas. $\mathrm{P}_{\mathrm{e} 1}$ and $\mathrm{P}_{\mathrm{e} 2}$ are the total gauge pressures of the co-axial jet and off-axial jet, which is varying from 1 bar to 4 bar. $\mathrm{Pa}$ is the ambient gauge pressure which is set to zero. The boundary condition of the workpiece-sapphire substrate is set to wall. Relationship between different processing parameters and removal rate of melt is studied by varying the $\mathrm{P}_{\mathrm{e} 1}, \mathrm{P}_{\mathrm{e} 2}$ and the intersection angle $(\theta)$ of the nozzles.

The computational domain shown in Fig. 2 is $75.0 \times 37.5 \times 5 \mathrm{~mm}^{3}$. Grid selection is an important factor to ensure the solution accuracy in most numerical simulation methods. Hence finite volume is meshed with unstructured mesh. The size of finite volume is $0.1 \mathrm{~mm}$ and the amount of finite volumes is 1727744 . In order to present the complex wave front of the jet, finite volumes should be remeshed by adaptive grid reconstruction method during the calculation process [18].

\section{Numerical results}

\subsection{Flow field of co-axial jet}

Fig.3 shows the contour of static pressure at different inlet pressure in the symmetry plane. The flow field of assisting gas jet is divided into free jet area, impingement area and wall jet area. When $\mathrm{P}_{\mathrm{e} 1}$ is $1 \mathrm{bar}$, there is hardly any shock in the flow field. Gas velocity is subsonic at the nozzle exit and static pressure in the wall jet area is almost 1 bar. There is not any pressure loss during the gas impinging on the substrate. When $\mathrm{P}_{\mathrm{e} 1}$ is 2 bar, radical gas expansion appears in the exit of co-axial nozzle obviously. Static pressure at nozzle exit drops below the standard atmospheric pressure. Due to the pressure of expansion area is less than the pressure in the free boundary, expansion wave will be reflected which develops compression wave. Under this circumstance, the compression wave is oblique shock which will reduce the static pressure in the wall jet area slightly. When inlet pressure is 3 bar, the flow behavior is similar to the one in 2 bar, but the position of oblique shock is closer to the substrate. When inlet pressure is 4 bar, the oblique shock disappears and a strong normal standoff shock is generated, which makes the flow velocity drop acutely and leads to a serious loss of energy and momentum. Fig.4 also shows the static pressure of wall jet area is only 2.3 bar when inlet pressure is 4 bar. As a result a high pressure inlet not only reduces static pressure but also generates vortex which finally decreases the absorption rate of laser and does harm to the melt materials removal.

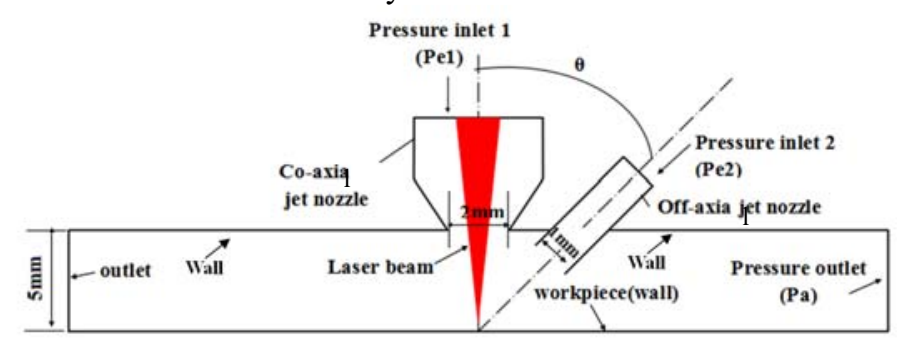

Fig.1 Model of twin gas jet-assisted laser scribing.

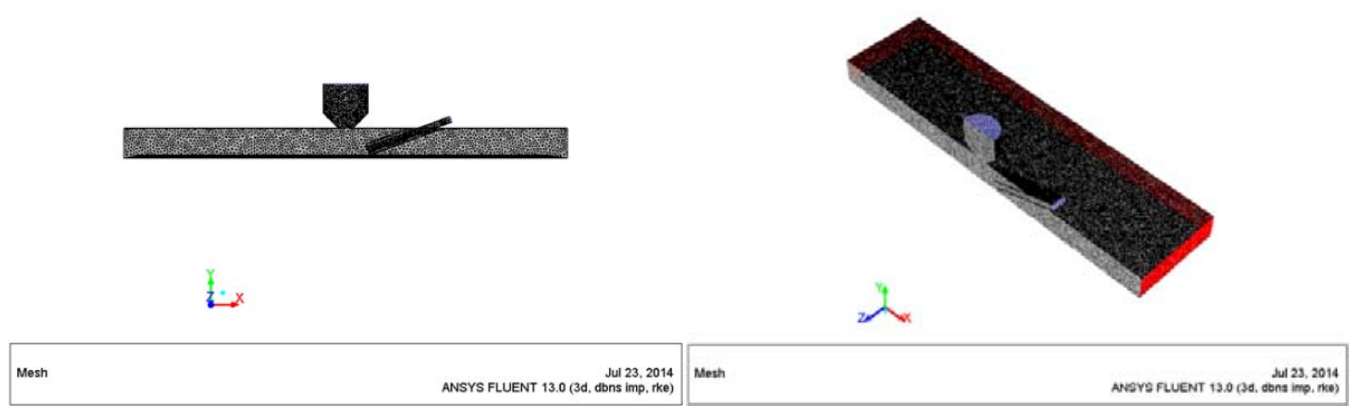

Fig.2 Grid structure of computation domain in the (a) cross section view (X-Y plane) and (b) axonometric view 


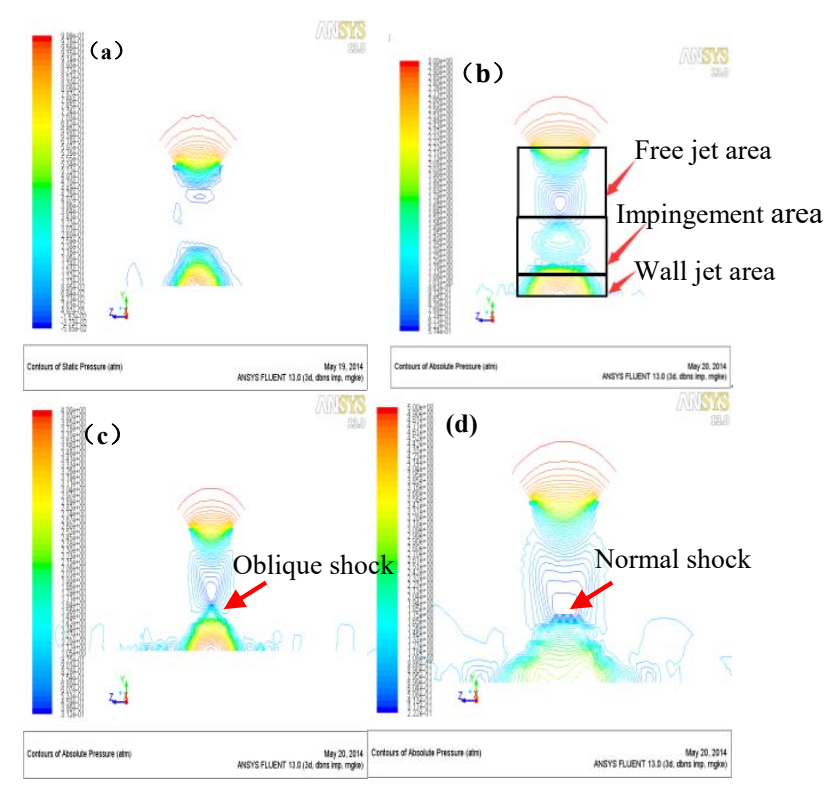

Fig.3 Contour of static pressure at different inlet pressure (a) 1 bar, (b) 2 bar, (c) 3 bar and (d) 4 bar.
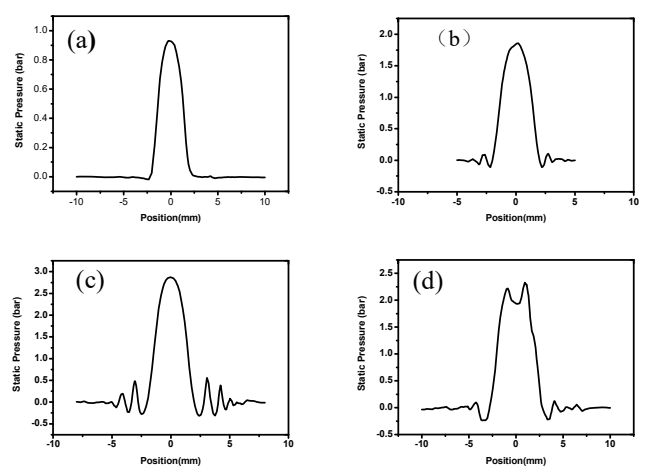

Fig.4 Static pressure in the groove when inlet pressure is (a) 1 bar,(b) 2 bar,(c) 3 bar and (d) 4 bar

\subsection{Flow field of off-axial jet}

Fig.5 shows the static pressure and dynamic pressure at laser irradiate region at different $\theta$ when co-axial inlet pressure is zero and off-axial inlet pressure is 2 bar. Analyzing the effect of off-axial jet, we suppose that the horizontal direction of off axis jet is the same with the laser scanning direction. When $\theta$ is less than $30^{\circ}$, static pressure in wall jet area is approximate 2 bar. When $\theta$ is larger than $30^{\circ}$, static pressure decreases with the increasing $\theta$. When intersection angle ascends to $85^{\circ}$, the static pressure gets the minimal value of 0.4 bar. It is difficult for impinging jet to remove the redeposited debris for the drag force is too low. On another hand, when intersection angle is less than $30^{\circ}$, dynamic pressure is about 0.2 bar. When $\theta$ is larger than $30^{\circ}$, dynamic pressure increases as the intersection angle grows. In other words, flow velocity and mass flow rate of assisting gas in the wall jet area increase with the increasing intersection angle.
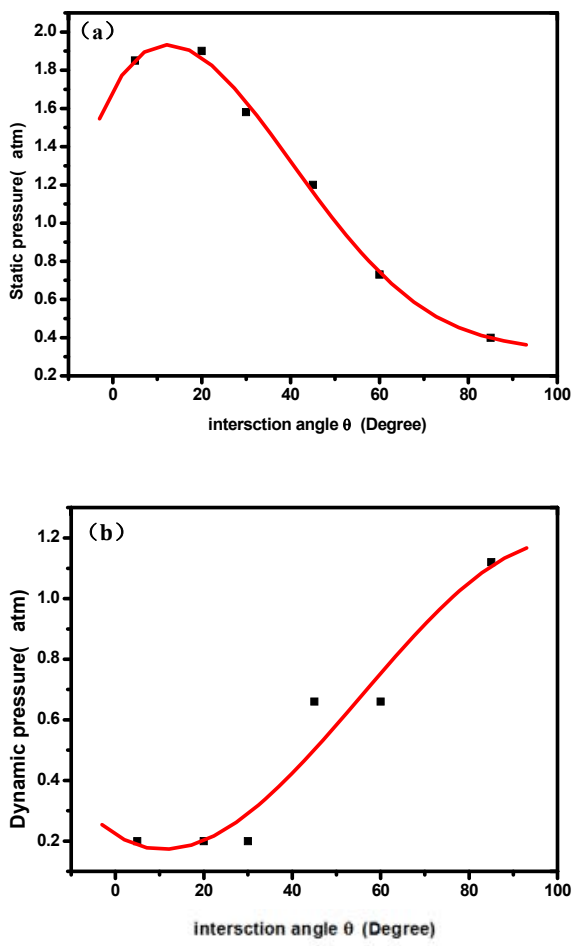

Fig.5 (a) Static pressure and (b) dynamic pressure at different $\theta$ when $\mathrm{P}_{\mathrm{e} 1}=0$ bar and $\mathrm{P}_{\mathrm{e} 2}=2$ bar

In order to improve the groove quality, it is necessary to ensure that mass flow rate is high enough to remove the melt material. That is to say, $\theta$ should be larger than $70^{\circ}$, which can ensure a high mass flow rate and reduce the loss of gas jet momentum in laser scribing. For another, a high intersection angle reduces the drag force to remove the melt material from the groove, which leads to a poorer cut quality. As a result, a too high or low intersection angle is not favorable for laser scribing. In order to enhance drag force and mass flow rate of assisting gas, intersection angle between co-axial and off-axial nozzle should be set to $70^{\circ}$.

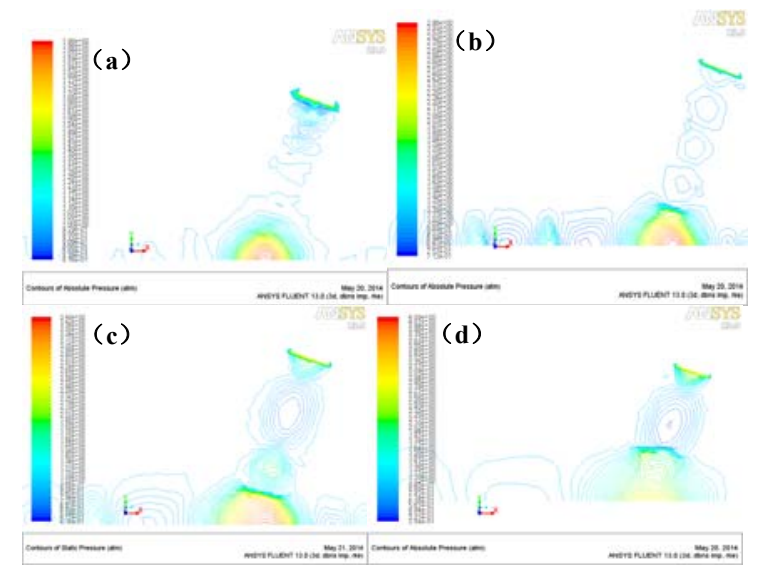

Fig.6 Contour of static pressure when off-axial inlet pressure is (a)1 bar,(b) 2 bar,(c) 3 bar and (d) 4 bar with $\theta$ of $70^{\circ}$ 


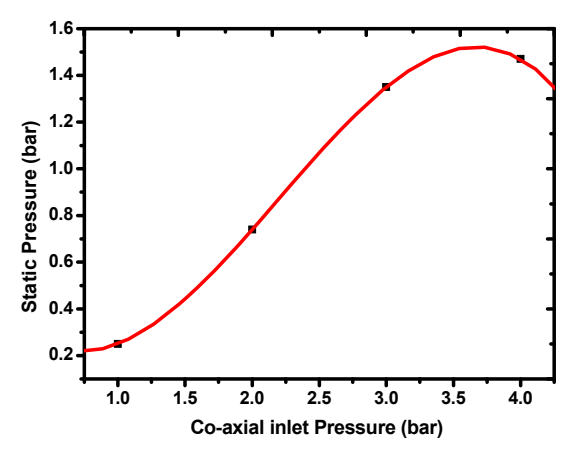

Fig.7 Static pressure at different off-axial inlet pressure when $P_{e 1}$ is 0 bar and $\theta$ is $70^{\circ}$

Fig.6 \& Fig. 7 show the contour of static pressure and the static pressure at different off-axial inlet presure when co-axial inlet pressure is zero and $\theta$ is $70^{\circ}$. There is no normal standoff shock appears with the increasing off-axial inlet pressure $\left(\mathrm{P}_{\mathrm{e} 2}\right)$. Obviously the static pressure increases with the increasing inlet pressure. However, static pressure rises gently when $P_{e 2}$ is higher than 3 bar. Because the type of off-axial nozzle is nonaxisymmetrical different from co-axial nozzle with convergent shape, it is difficult to generate the normal standoff shock. Therefore flat fan nozzle is better than the convergent nozzle in laser scribing. But it is still difficult to increase static pressure when $\mathrm{P}_{\mathrm{e} 2}$ is more than 3 bar, because the shock reflection effect of air weakens the kinetic energy of the assisting gas which makes a considerable total pressure loss. Therefore the ideal off-axial inlet pressure is about 3 bar.

\subsection{Flow field of twin assisting gas}

Fig.8 shows the velocity vector of flow field at different $\theta$ when both co-axial and off-axial inlet pressure are 2 bar. When $\theta$ is smaller than $45^{\circ}$, coaxial gas jet and off-axial gas jet crash and reflect above the laser irradiate region, which causes a high pressure area in the wall jet area where gas velocity and mass flow rate is extremely low. As the intersection angle $\theta$ increases, stagnation area disappears and the flow velocity and mass flow rate increase. As a result it is easier to remove the melt material from the groove at this circumstance. When $\theta$ is higher than $70^{\circ}$, off-axial jet gas collides with the reflected gas jet of co-axial jet, which decreases the total pressure acutely. Therefore excessive high intersection angle causes a great total pressure loss, which not only reduces the drag force of assisting gas but also reduces the mass flow rate in the wall jet area. Therefore, the optimum intersection angle is around $70^{\circ}$.

Fig.9 and Fig.10 show the velocity vector and static pressure of the flow field at different inlet pressure combination respectively. When $\mathrm{P}_{\mathrm{e} 2}$ is smaller than 3 bar, the collision and reflection of the two assisting gas is slight, which can obtain an ideal mass flow rate in laser scribing. When $\mathrm{P}_{\mathrm{e} 2}$ is 4 bar, a strong collision and reflection occur and mass flow rate decrease. It means that a great loss of energy and momentum will occur if $\mathrm{P}_{\mathrm{e} 2}$ exceeds 3 bar. That is why the static pressure is higher than other inlet pressure combinations when $\mathrm{P}_{\mathrm{e} 1}=2$ bar and $\mathrm{P}_{\mathrm{e} 2}=3$ bar. In the same way, excessive co-axial inlet pressure blocks the flow of off-axial jet and reduces the total pressure in the wall jet area. To prevent the furious collision of the two impinging jet, co-axial inlet pressure should be reduced appropriately. Therefore the optimum inlet pressure combination is the one co-axial inlet pressure of 2 bar and off-axial inlet pressure of 3 bar.

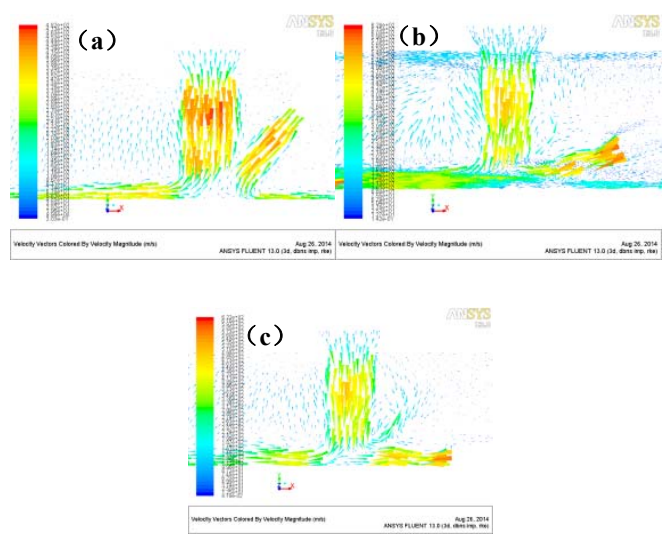

Fig.8 Velocity vector of flow field at different $\theta$ (a) $45^{\circ}$, (b) $70^{\circ}$ and (c) $85^{\circ}$ when inlet pressure $\mathrm{P}_{\mathrm{e} 1}=\mathrm{P}_{\mathrm{e} 2}=2$ bar.

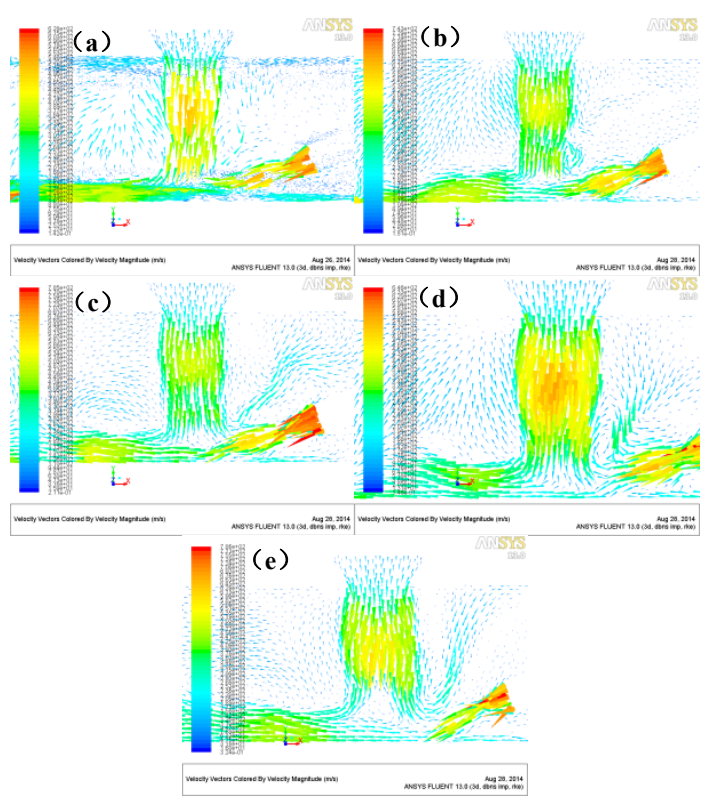

Fig.9 Velocity vector of flow field at different inlet pressure combination (a) $\mathrm{P}_{\mathrm{e} 1}=2$ bar , $\mathrm{p}_{\mathrm{e} 2}=2 \mathrm{bar}$; (b) $\mathrm{P}_{\mathrm{e} 1}=2$ bar, $\mathrm{P}_{\mathrm{e} 2}=3$ bar; (c) $\mathrm{P}_{\mathrm{e} 1}=2$ bar, $\mathrm{P}_{\mathrm{e} 2}=4$ bar; (d) $\mathrm{P}_{\mathrm{e} 1}=3$ bar, $\mathrm{P}_{\mathrm{e} 2}=3$ bar; (e) $\mathrm{P}_{\mathrm{e} 1}=4$ bar, $\mathrm{p}_{\mathrm{e} 2}=4$ bar. 


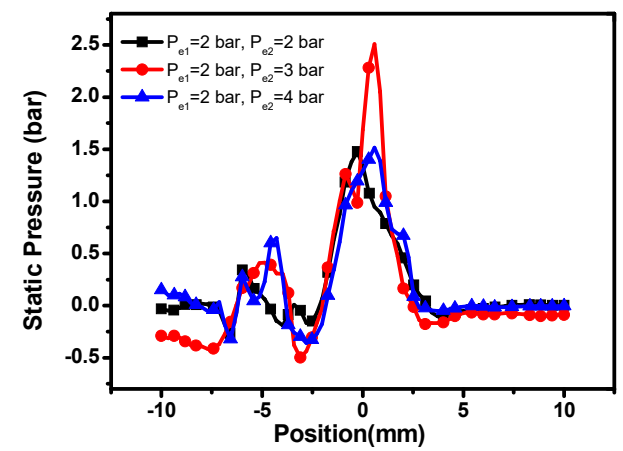

Fig.10 Static pressure on the centerline of co-axial nozzle at different inlet pressure combination

(a) $\mathrm{P}_{\mathrm{e} 1}=2$ bar, $\mathrm{P}_{\mathrm{e} 2}=2$ bar; (b) $\mathrm{P}_{\mathrm{e} 1}=2$ bar, $\mathrm{P}_{\mathrm{e} 2}=3$ bar; (c) $\mathrm{P}_{\mathrm{e} 1}=2$ bar, $\mathrm{P}_{\mathrm{e} 2}=4$ bar.

\section{Experiments}

\subsection{Experimental facility}

A $532 \mathrm{~nm}$ DPSS (Diode Pumped Solid State) laser (Lightwave, Series 210G) at a pulse duration of $45 \mathrm{~ns}$ is used as a light source of laser processing system to verify the simulation results. Its spot diameter can be adjusted at the range of $10 \sim 20 \mu \mathrm{m}$. In order to protect the focusing lens and remove the melt material, co-axial and off-axial assisting gases are used with inlet pressure ranged from 0 to 4 bar. The structures of the nozzles are the same with the one in the simulation. Horizontal direction of the off axis jet is the same with the laser scanning direction. The translations in the $x / y / z$ directions and the rotation in the $\mathrm{x}-\mathrm{y}$ plane are controlled by the computer generated signals. The pulse repetition frequency used in this experiment is fixed at $1 \mathrm{kHz}$. The substrate is the single side polishing single crystal sapphire substrate with dimension of 2 inch $\times$ $432 \mu \mathrm{m}$ (diameter $\times$ thickness). The groove width and depth are measured by optical microscope (Zxiss AX10) and laser scanning confocal microscope (Olympus OLS4000). The front surface and section of the groove are observed by laser scanning confocal microscope and environmental scanning electron microscopy (FEI Quanta 400 FEG).

\subsection{Influence of co-axial jet}

Fig.11-12 shows the relationship between coaxial inlet pressure and the groove depth \& width at different laser pulse energy and scanning velocity. We can know that higher $\mathrm{P}_{\mathrm{e} 1}$ leads to the shallower groove when the laser pulse energy is $150 \mu \mathrm{J}$ and the scanning velocity is $1 \mathrm{~mm} / \mathrm{s}$. However, when the scanning velocity is high $(>1 \mathrm{~mm} / \mathrm{s})$, the difference is small in groove depth at different inlet pressure. The reason is that when the scanning velocity is $1 \mathrm{~mm} / \mathrm{s}$, the assisting gas cools the surface of substrate, which reduces the absorption of laser and decreases groove depth when the scanning velocity is $1 \mathrm{~mm} / \mathrm{s}$. When the pulse energy is high $(650 \mu \mathrm{J})$, the difference in groove depth is minor with the increasing the inlet pressure at different scanning velocity. The reason is that laser energy absorption is much higher than the one assisting gas carried off. As a result, there is little difference at different inlet co-axial pressure.

When laser pulse energy is low $(150 \mu \mathrm{J})$, groove width increases with the increasing co-axial inlet pressure. This phenomenon will become more obvious when scanning velocity is lower $(1 \mathrm{~mm} / \mathrm{s})$. Then more melting materials deposits and adheres to the groove edge which enlarges the groove width. When laser pulse energy is high $(650 \mu \mathrm{J})$ and scanning velocity is low $(1 \mathrm{~mm} / \mathrm{s})$, groove width also increases with the increasing inlet co-axial pressure. However, when laser pulse energy is high $(650 \mu \mathrm{J})$ and scanning velocity is high $(\geq 4 \mathrm{~mm} / \mathrm{s})$, groove width shows a trend from decline to rise and reaches the minimum at an inlet pressure of 3 bar. It matches the simulation result because when inlet pressure is about 3 bar, the static pressure in the wall jet area get the maximal value, which can offer the largest drag force to carry out the melt material from the groove. When the inlet pressure is larger than 3 bar, normal standoff shock wave will be generated. It results in the scattered assisting gas which will reduce the static pressure and the ability to remove the melt. In brief, similar to the result of chapter 3.1, the optimal coaxial inlet pressure is in the range of 2-3 bar.
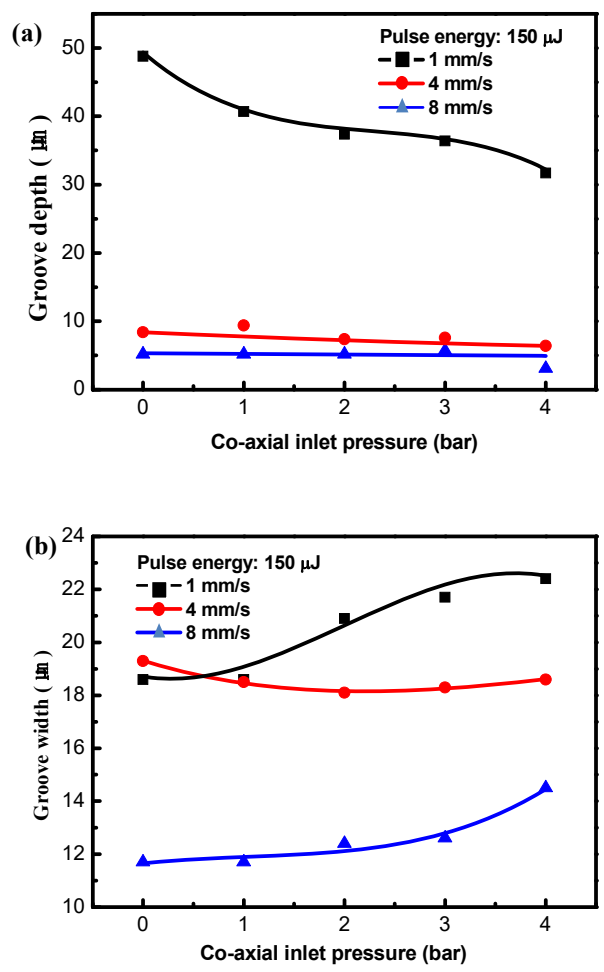

Fig.11 Groove width and groove depth at different coaxial inlet pressure when pulse energy is $150 \mu \mathrm{J}$ 

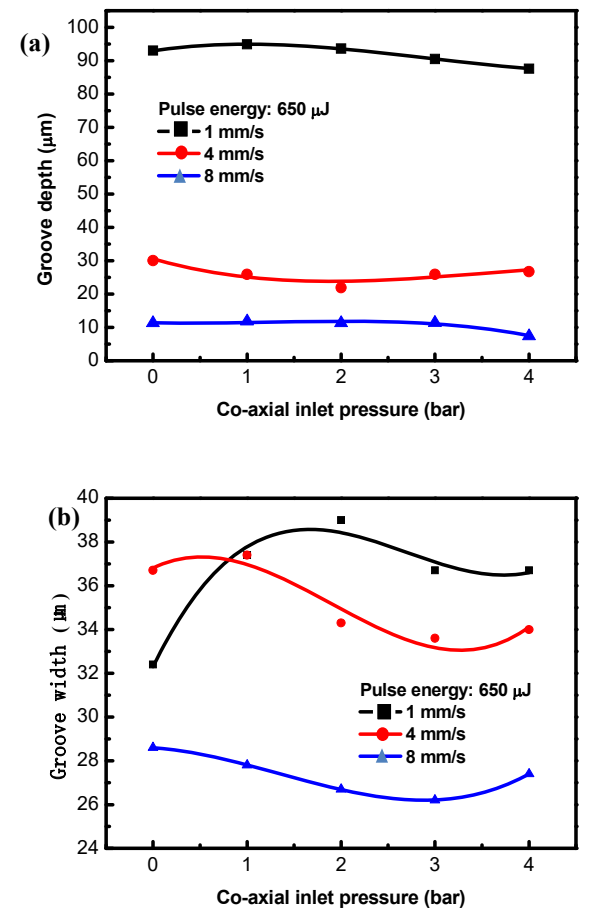

Fig.12 Groove width and groove depth at different co-axial inlet pressure when pulse energy is $650 \mu \mathrm{J}$

\subsection{Influence of off-axial jet}

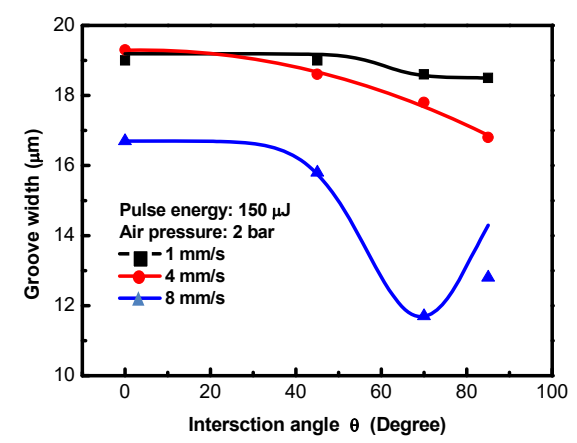

Fig.13 Groove width at different $\theta$ when pulse energy is $150 \mu \mathrm{J}$, co-axial inlet pressure of $0 \mathrm{bar}$, off-axial inlet pressure of 2 bar and $\theta$ of $70^{\circ}$

Fig.13 shows the relationship between the intersection angle and the groove width. If $\theta$ is smaller than $45^{\circ}$ with $\mathrm{P}_{\mathrm{e} 1}=0$ bar $\& \mathrm{P}_{\mathrm{e} 2}=2$ bar. When $\theta$ is higher than $45^{\circ}$, groove width decreases with the increasing intersection angle. While $\theta$ reaches $70^{\circ}$, the groove width reaches the minimum. The reason of this phenomenon is that dynamic pressure in wall jet area is less than 0.3 bar when $\theta$ is smaller than $45^{\circ}$. When intersection angle is higher than $45^{\circ}$ dynamic pressure rises rapidly. This greatly improves the mass flow rate and enhances the melt removal capability. The reason why groove width does not decrease anymore when intersection angle is higher than $70^{\circ}$ is that an exorbitant angle leads to a low drag force, which will hinder the melt removal. Front surfaces of

groove can be observed in Fig. 14. When $\theta$ is $70^{\circ}$, the groove edge is flatter than other conditions. In conclusion, the best intersection angle between coaxis and off-axis is around $70^{\circ}$.
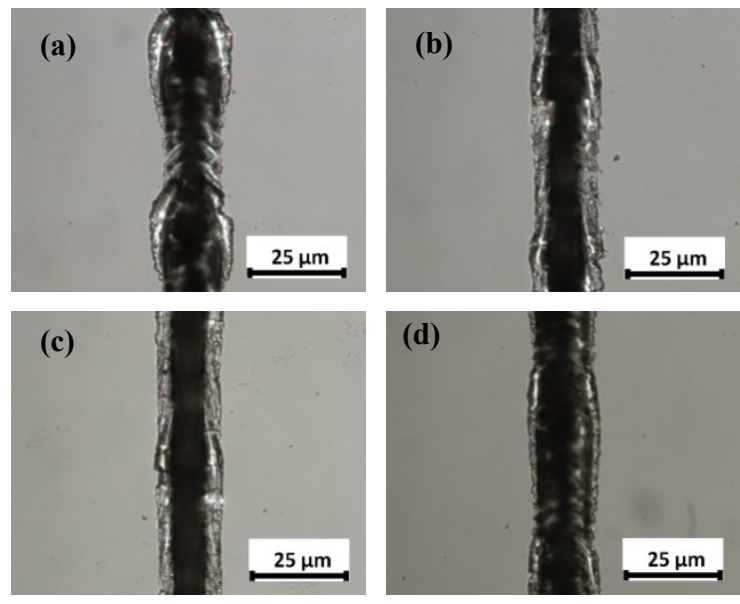

Fig.14 Front surfaces of the groove at different $\theta$ (a) without any assisting gas, (b) $\theta=45^{\circ}$, (c) $\theta=70^{\circ}$ and (d) $85^{\circ}$ when pulse energy is $150 \mu \mathrm{J}$,scanning velocity of $8 \mathrm{~mm} / \mathrm{s}$, $\mathrm{P}_{\mathrm{e} 1}=0$ bar and $\mathrm{P}_{\mathrm{e} 2}=2$ bar

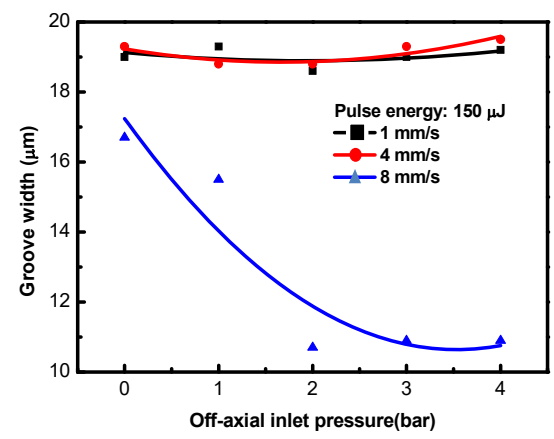

Fig.15 Groove width with different off-axial inlet pressure at different scanning velocity when pulse energy is $150 \mu \mathrm{J}$
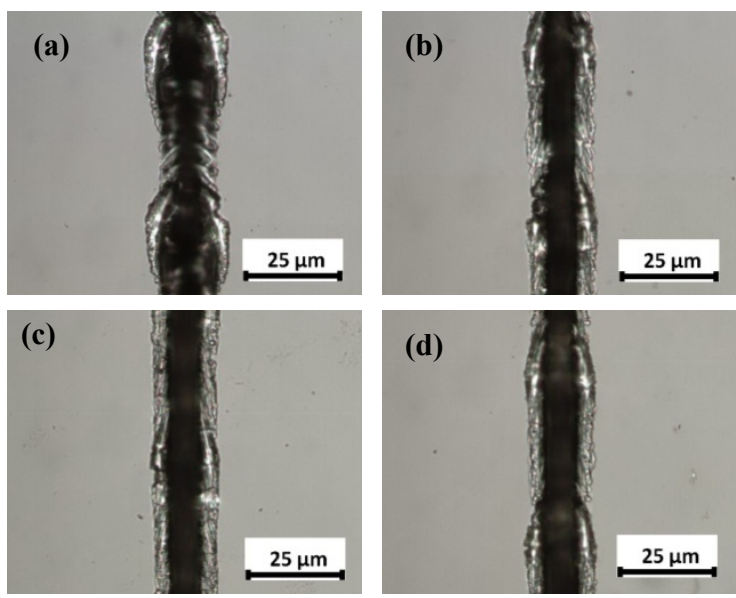

Fig.16 Front surfaces of groove at different off-axial inlet pressure (a) 0 bar, (b) 1 bar, (c) 2 bar, (d) 3 bar when pulse energy is $150 \mu \mathrm{J}$ and scanning velocity is $8 \mathrm{~mm} / \mathrm{s}$ 
Fig. 15 shows the groove width as a function of off-axial inlet pressure. The groove width decreases with the increasing off-axial inlet pressure and saturates when off-axial inlet pressure is higher than 3 bar. This is because if inlet pressure is lower than 3 bar, static pressure increases with the increasing inlet pressure. However static pressure does not increase further when inlet pressure is higher than 3 bar. The optimal off-axial inlet pressure should be 2 3 bar which is also verified from the front surfaces of the groove, shown in Fig. 16.

\subsection{Influence of twin assisting gas}

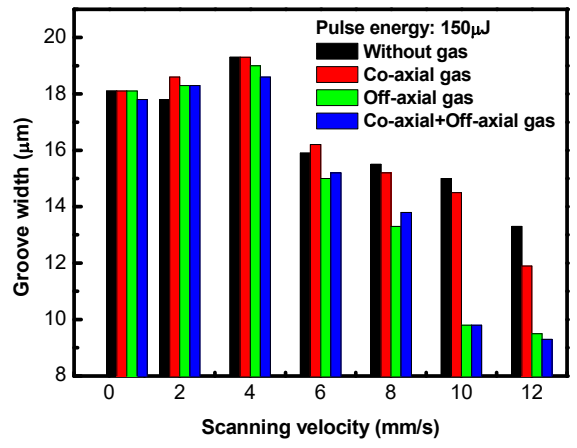

Fig.17 Groove width at different scanning velocity and different assisting gas condition when inlet pressure is 2 bar

Fig.17 shows the groove width with different scanning velocity under different assisting gas conditions. It is clear that in most cases a smaller groove width can be obtained when using the offaxial assisting gas and twin assisting gas.

Fig18 shows the front surfaces of groove under the assisting gas conditions of without assisting gas, co-axial assisting gas only, off-axial assisting gas only and twin assisting gas respectively. The melt material can't be effectively removed from the groove and adheres on the edge which magnifies the groove width if no assisting gas is used. When using co-axial assisting gas, the groove width is slightly smaller than the one not using any assisting gas, but the groove edge is still rough. The groove width reduces and the groove quality improves when using off-axial assisting gas. When using twin assisting gas both in co-axis and off-axis, an optimum groove quality will be obtained, which has a flat groove edge without adhering a lot of redeposition layer.

Although drag force to carry out the melt material is larger enough when only using co-axial assisting gas, the mass flow rate is still too small to remove the melt material since the velocity of the jet is still small in the laser irradiate region. Without enough mass flow rates the strong pressure will compact the melt material and make it difficult to eliminate. By adding off-axial assisting gas which accelerates the gas velocity in the wall jet area, an ideal assisting gas jet with high drag force and high mass flow rate will remove the melt material from the groove efficiently.
In order to acquire a high quality groove in pulsed green laser sapphire scribing, it is necessary to use assisting gas jet both in co-axial nozzle and off-axial nozzle.

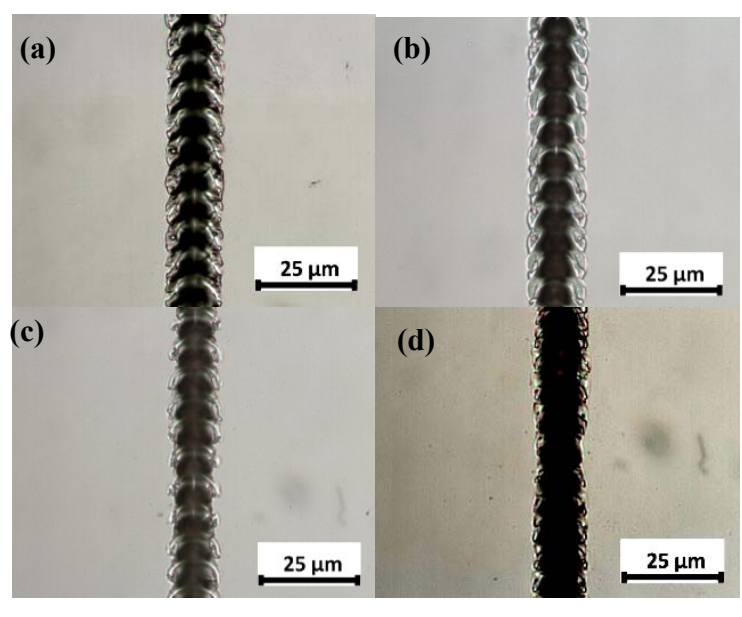

Fig.18 Front surfaces of groove at different condition of assisting gas (a) without assisting gas, (b) co-axial assisting gas only, (c) off-axial assisting gas only and (d) twin assisting gas when pulse energy is $150 \mu \mathrm{J}$, scanning velocity of $8 \mathrm{~mm} / \mathrm{s}$ and $\mathrm{P}_{\mathrm{e} 1}=\mathrm{P}_{\mathrm{e} 2}=2$ bar.

\section{Conclusions}

The laser scribing capacity or dross-removal capability is determined by the drag force and the mass flow rate of the assisting gas. The melt material can be carried out from the groove efficiently under a high level jet drag force and mass flow rate. The coaxial gas jet provides a high drag force while the offaxial gas jet improves the mass flow rate in laser sapphire scribing. To optimize the groove quality it is necessary to use the twin assisting gas both in co-axis and off- axis.

When using the co-axial assisting gas jet in laser sapphire scribing, the co-axial inlet pressure should not be set to a high value because higher inlet pressure ( $>3$ bar) of convergent nozzle results in the generation of normal stand-off shock. It will reduce the static pressure in laser irradiate region and disrupt the gas flow drastically. Therefore, the optimum coaxial inlet pressure is 2 bar in order to maximize the drag force of assisting gas and avoid the generation of normal stand-off shock.

Only using the co-axial assisting gas can not remove the melting material efficiently because the mass flow rate is still too small in this way. Using off-axial assisting gas can accelerate the gas velocity in laser irradiate region and remove the redeposited debris and avoid the redeposited debris with sufficient gas flow with sufficient mass flow rate. The ideal intersection angle $(\theta)$ and inlet pressure of off-axial assisting gas is $70^{\circ}$ and $2 \sim 3$ bar respectively.

Using twin assisting gas both in co-axis and offaxis can provide a high drag force and mass flow rate which improves the groove quality and optimizes the groove size in pulsed green laser sapphire scribing. 
When co-axial inlet pressure is 2 bar, off-axial inlet pressure of 2 3 bar, intersection angle $(\theta)$ of $70^{\circ}$ and laser pulse energy of $150 \mu \mathrm{J}$, a good groove quality and size will be obtained.

\section{Acknowledgments}

Financial assistance for this work is granted by the National Nature Science Foundation of China (No.51575114 \& 51275096), Guangdong Natural Science Foundation (No.S2013010014070) and Ordinary University Characteristics Innovation Project of Guangdong Province (Natural Science, No.2014KTSCX059).

\section{References}

[1] Elena R. Dobrovinskaya, Leonid A. Lytvynov, and Valerian Pishchik: "Sapphire: Material, Manufacturing, Applications", (Springer, New York, 2009) p.233.

[2] K. Chen, Y. Lawrence Yao, and V. Modi: J. Manuf. Sci. E.-T. ASME., 122, (2000) 429.

[3] W. O'Neill, M. Voglsanger, A. Elboughey, and W.M. Steen: P. I. Mech. Eng. B-J. Eng., 215, (2001) 1051.

[4] K.H. Sezer, L. Li, and S. Leigh: Int. J. Mach. Tool Manu., 49, (2009) 1126.

[5] C.C. Mai, and J. Lin: Opt. Laser Technol., 34, (2002) 479.

[6] O.A. Melhem, B.S. Yilbas, and S.Z. Shuja: Opt. Laser Eng., 49, (2011) 384.

[7] B. Tirumala Rao and A.K. Nath: SadhanaACAD. P. Eng. S., 27, (2002) 569.

[8] H.J. Chen, W.Y. Lin, and Y.J. Chang: Int. J. Adv. Manuf. Tech., 79, (2015) 449.

[9] S.G. Guo, J. Hu, and L. Luo: Appl. Laser, 27, (2007) 403.

[10] J. Hu, S.G. Guo, L. Luo, Z.Q. Yao: Int. J. Adv. Manuf. Tech., 39, (2008) 716.

[11] M.Y. Qiu and J. Hu: Chin. J. Lasers, 5, (2009) 1296.

[12] F. Al-Sulaiman, B.S. Yilbas, M. Ahsan and C. Karates: Int. J. Adv. Manuf. Tech., 45, (2009) 62.

[13] S. Elhadj, M.J. Matthews, G.M. Guss, and I.L. Bass: Appl. Phys. B-Lasers O., 113, (2013) 307.

[14] V. Yakhot and S.A. Orszag: S. Sci. Comput., 1, (1986) 1.

[15] V. Yakhot and S.A. Orszag: Phys. Fluids, A4, (1992) 1510.

[16] V. Yakhot and S.A. Orszag: J. Sci. Comput., 1, (1991) 39.

[17] A.D. Brandt and G.S. Settles: J. Laser Appl., 3 (1997) 269.

[18] J. Xu and C. Zhao: Int. J. Heat. Mass. Tran., 50, (2007) 2434.

(Received: May 24, 2015, Accepted: April 6, 2016) 